\title{
Characterization of E-beam Fabricated Gold Nanoparticles
}

\author{
Steven Madsen, Paul Kempen, Robert Sinclair
}

Stanford University, Department of Materials Science and Engineering, Stanford, CA 94305-4034, USA.

Surface Enhanced Raman Spectroscopy (SERS) is a powerful technique for detection of small concentrations of molecules which makes use of inelastic scattering of light near nanostructured metal surfaces. It has been widely proposed that the scattering efficiency is improved by a local enhancement in the electric field from surface plasmons in the noble metal particles [1]. In spite of this, there has been no definitive correlation between localized surface plasmon resonances, SERS enhancement and morphology. [2]

The approach herein outlines a procedure to produce repeatable and adjustable arrays of metallic nanoparticles via electron beam lithography for analysis by optical and electron techniques. The procedure allows for controlled adjustment of particular morphological feature while holding all others constant. By modifying a single feature at a time, the effect on both plasmon frequency and SERS intensity can be established.

Synthesis is performed using a JEOL 6300 electron beam (e-beam) lithography tool. This procedure uses an electron beam to trace out a programmed pattern on a layer of poly(methyl methacrylate) [an electron beam sensitive positive resist] with $8 \mathrm{~nm}$ resolution, which is then developed into a mask for nanoparticle deposition. The latter are generated by evaporating a $2 \mathrm{~nm}$ Cr layer followed by 20 to $30 \mathrm{~nm}$ of Au onto the substrate and lifting off the mask by dissolving the remaining PMMA in acetone. This process gives control over not only nanoparticle size and shape, but also orientation of the nanoparticles and periodicity of the array.

Both SEM and AFM imaging were carried out. Characterization is important to assess the quality of the lithography and the repeatability of the synthesized structures. SEM imaging was performed using an FEI Magellan 400 XHR. Images were taken at 3 to $25 \mathrm{kV}$ and 25 to $100 \mathrm{pA}$ current. Figure 1 shows a series of SEM images of disk shaped nanoparticles fabricated with this technique. The grain structure is visible in the nanoparticles, demonstrating that they are polycrystalline.

Two hundred disk shaped nanoparticles were measured from SEM images to determine the control and reproducibility of patterning. The radius averaged $73 \mathrm{~nm}$ with a standard deviation of $3 \mathrm{~nm}$, compared to an aim of $74 \mathrm{~nm}$. In another study using equilateral triangular nanoparticles (Figure 2) of side length $72 \pm 4 \mathrm{~nm}(75 \mathrm{~nm}$ aim), corner radii were measured at $6 \pm 2 \mathrm{~nm}$. Nanoparticle gap sizes below $20 \mathrm{~nm}$ were consistently achievable.

AFM was used to characterize the height of the nanoparticles. While convolution of the tip and the nanoparticle reduces in-plane resolution, AFM is a widely used tool for height mapping. The AFM results (Figure 3) show an average height difference from peak to trough of $33 \mathrm{~nm}$, which is very close to the aim of $2 \mathrm{~nm} \mathrm{Cr}$ plus $30 \mathrm{~nm} \mathrm{Au}$.

This study demonstrates that electron beam lithography, as expected, is a powerful technique for 
creating arrays of tunable nanoparticles, with controlled pitch and orientation relative to one another, in order to gain a better understanding of the relationship between nanoparticle structure and SERS properties. Subsequent work includes deposition of nanoparticles directly onto TEM grids for analysis of the localized surface plasmons by electron energy loss spectroscopy [3]. This work sets the foundation for this type of fabrication and characterization.

\section{References:}

[1] J. Ferarro in "Introductory Raman Spectroscopy”, (Academic Press, San Diego) p. 160-163.

[2] N. Mirsaleh-Kohan, et al. J. Phys. Chem. Lett. 3 (2012), p. 2303-2309.

[3] A.L. Koh et al, Nano Letters, 11 (2011), p. 1323-1330.

[4] This research is supported by the Center for Cancer Nanotechnology Excellence and Translation (CCNE-T) grant funded by NCI-NIH to Stanford University U54CA151459.

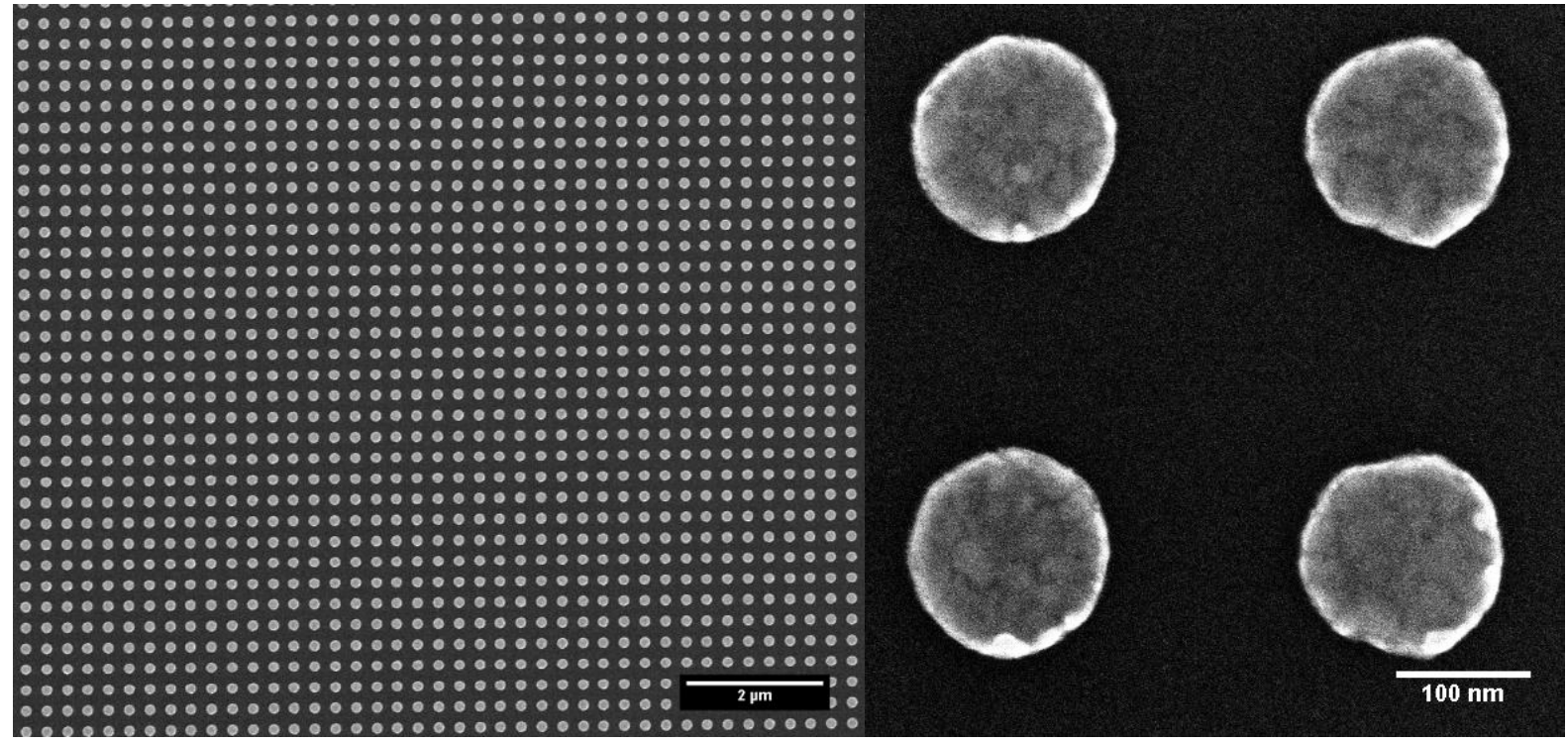

Figure 1. Low and high magnification SEM images of an array of Au disks fabricated by e-beam lithography.

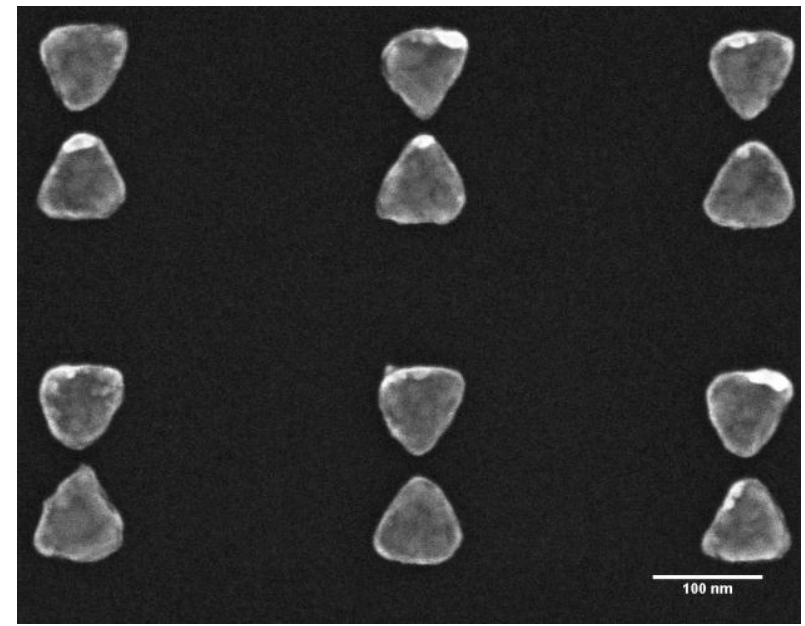

Figure 2. Array of "bow-tie" nanoparticles showing small gaps between them.

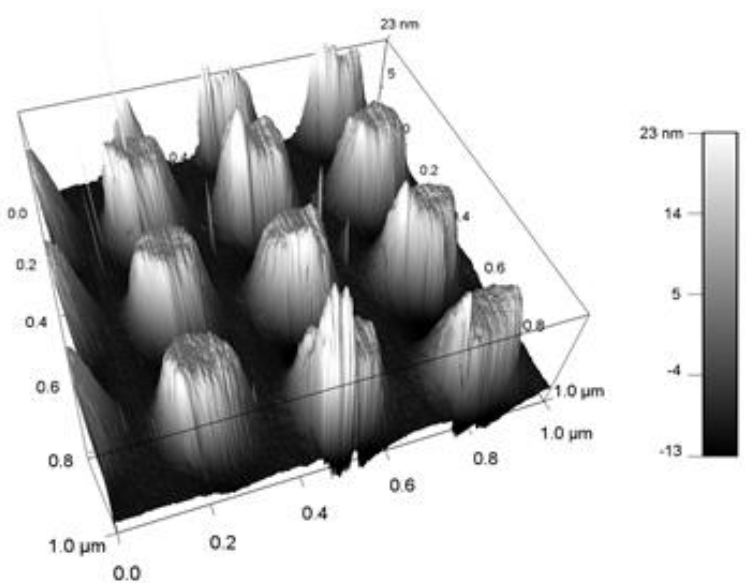

Figure 3. AFM image used to measure particle height. Greyscale colormap shows relative height in $\mathrm{nm}$ of a $1 \mu \mathrm{m} \times 1 \mu \mathrm{m}$ section of particles. 